\title{
Temps pascal ou L’horizontalité religieuse de la paternité symbolique
}

\author{
François Ouellet \\ Université du Québec à Chicoutimi
}

Ici on applique la justice, pas la loi

- Temps pascal

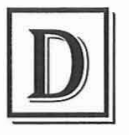

ans le cadre de cet article, je me propose de poursuivre le travail d'investigation sur la représentation de la figure du Père dans l'œuvre de Daniel Poliquin, que j'ai amorcé il y a quelques années ${ }^{1}$, en traitant du premier roman de l'auteur, Temps pascal. On verra notamment comment ce roman ouvrait la voie à la complexité narrative et discursive du roman suivant, L'Obomsawin, auquel j'ai récemment consacré une étude².

La première image de toute l'œuvre de Poliquin (l'incipit de Temps pascal) est celle d'un homme défait, qui a lutté de peine et de misère contre une envie suicidaire. Vingt ans plus tôt, Médéric Dutrisac, ancien maire communiste et syndicaliste de renom, s'était retiré de toute action politique pour vivre isolé dans une forêt du nord de l'Ontario. Or, au moment où le roman commence, il traverse une sévère dépression, qui l'a amené à vouloir

1 Voir notamment F. Ouellet, "Se faire Père. L'œuvre de Daniel Poliquin ", dans La littérature franco-ontarienne : enjeux esthétiques, sous la dir. de L. Hotte et F. Ouellet, Ottawa, Le nordir, 1996, p. 91-116. "Père " s'entend comme un signifiant qu'il ne faut pas confondre strictement avec le " père de famille » de la réalité romanesque. Le « Père » comme signifiant participe d'une dynamique textuelle qui confronte le héros aux figures d'autorité que peuvent être aussi bien la Loi, l'Église, l'Institution, etc.

2 Voir F. Ouellet, "L'Obomsawin ou l'impossible paternité ", Voix et images, vol. XXVIII, no 3, printemps 2002, p. 448-460. Le présent article s'inscrit dans le cadre d'une recherche subventionnée par le CRSH. 
se laisser mourir. Pour réagir, il marche depuis deux jours et deux nuits dans le bois enneigé, avec raquettes et boussole, afin de rejoindre "la grand-route » qui mène vers Subdury, où il espère pouvoir se faire examiner par un médecin. Heureusement, Médéric, ayant enfin atteint la route, est recueilli par un autobus. Voilà donc la première image de Temps pascal. En contrepartie, voici l'excipit du dernier roman de Poliquin, L'homme de paille : Benjamin Saint-Ours, qui, après avoir quitté la Nouvelle-France, a vécu au Labrador avec les Esquimaux, dérive " vers l'inconnu » sur une banquise dans l'Arctique.

Dans les deux cas, nous avons d'abord l'image de personnages en fuite, qui espèrent que de leur situation difficile puisse naître une vie nouvelle. Mais tandis que Benjamin caresse l'espoir un peu fou de donner forme à "l'homme nouveau " qui se forme en lui, puisqu'on ne voit pas tellement comment il pourrait s'en sortir ${ }^{3}$, Médéric est sur le point, à la suite de la rencontre qu'il fera dans l'autobus qui l'a recueilli, de tenter de refaire sa vie. Ces personnages en fuite qui veulent faire peau neuve nous font penser, bien sûr, à la légende de l'écureuil noir, qui ne signifie pas autre chose que cette volonté de nouveau départ. Dans L'écureuil noir, Calvin Winter fait publier sa propre notice nécrologique; il est inspiré, dans son désir de changer de vie, par d'anciens rats qui, pour échapper à des mesures de dératisation, se seraient mêlés à des écureuils gris. Devenus des écureuils noirs, ils n'auraient principalement conservé de leur passé que la couleur de leur pelage. Il n'est pas sans intérêt de savoir que Poliquin a développé cette légende " en début de carrièré » (une dizaine d'années avant qu'il ne la

3 Mais Poliquin, qui se décrit comme un "optimiste", préfere penser que cet espoir sera comblé : «Moi, je suis un optimiste par tempérament. La banquise a peut-être fini par fondre, et il [Benjamin] a profité d'un courant de vent pour aboutir en Écosse. Et de là il a gagné le continent. Je suis assez renseigné en matière maritime pour savoir que c'est possible. Logiquement, cependant, il est mort : le glaçon s'est ouvert et Benjamin a sombré dans le tourbillon qui a suivi. Mais dans ma vision du monde, pleine d'espérance, non, l'homme est combatif, il conjure la mort. " (F. Ouellet, "Le roman de "l'être écrivain" entre l'anonymat et la reconnaissance. Entretien avec Daniel Poliquin ", Voix et images, vol. XXVIII, $\mathrm{n}^{\circ} 3$, printemps 2002, p. 410. Cette impasse dans laquelle se trouve le personnage et l'ambiguïté que lui donne le commentaire de l'auteur me semblent tout à fait significatifs de la tension fondamentale qui habite, dans l'œuvre de Poliquin, la représentation du signifiant paternel dont je traite dans le présent article.

4 D. Poliquin, "Note de l'auteur " qui précède la nouvelle "Pourquoi les écureuils noirs d'Ottawa sont noirs ", (D. Poliquin, Nouvelles, préface de 
récupère dans le cadre de son roman), car, de fait, elle figure depuis comme l'espèce de socle discursif sur lequel l'ensemble de l'œuvre parait se fonder, l'auteur donnant en quelque sorte à reconnaître cette légende d'un texte à l'autre sous diverses formes métaphoriques, qu'il s'agisse de la réincarnation que vise l'héroïne de la nouvelle "Avoir su " (dans Le Canon des Gobelins) ou des « retours à la vie » de Benjamin Saint-Ours, qui a d'abord passé sept ans dans le coma, puis qui a dormi cinq autres années. En outre, cette légende, de laquelle découlent les métaphores des romans subséquents, s'est d'emblée conceptualisée en fonction de la représentation signifiante du père. Ce n'est pas sans raison si Calvin Winter prend la décision de changer d'identité immédiatement après avoir débranché l'appareil qui maintenait son père en vie. Le " meurtre du père " permet au fils de reconfigurer les liens identitaires et de repenser la paternité symboliques. Dans "Pourquoi les écureuils noirs d'Ottawa sont noirs ", il s'agit de " changer de peau " afin d'assurer l'héritage et la filiation. Le narrateur argue : "Nous n'avons pas le choix. Mourir rat ou vivre écureuil. Voir notre tribu, nos enfants, s'éteindre sans lendemain. Ou alors, prendre les devants : se réincarner tout de suite, se réinventer en une nouvelle race, pour assurer à chacun, ainsi qu'il le mérite, le salut individuel ! Moi, Wilfrid, votre archonte, j'ai décidé de vivre et je vous invite à me suivre dans cette voie nouvelle $!^{6}$ "

La question du père est centrale dans l'œuvre de Poliquin, et c'est encore elle — on s'en doute - qui détermine fondamentalement la posture des personnages dans l'incipit de Temps pascal et dans l'excipit de L'homme de paille. Le destin auquel aspirent Médéric Dutrisac et Benjamin Saint-Ours, dont l'espérance les oblige chaque fois à chercher à refaire leur vie pour pouvoir tenir les promesses de ce destin, c'est celui de la figure d'autorité du père symbolique. Disons-le de la façon la plus simple possible : il suffirait que le héros de Poliquin devienne symboliquement père (par opposition à biologiquement père) pour qu'il soit comblé et qu'il puisse assumer sa vie au lieu de chercher à la recommencer. Chaque roman de Poliquin traite la question de manière différente, mais l'enjeu reste toujours le même (accéder au rang de père), et le résultat est invariablement décevant (il y a échec). Entre le début de Temps pascal, qui présente, nous le verrons, l'image d'un père déchu, et la fin de L'homme de paille, qui

F. Ouellet, Ottawa, Le Nordir, coll. " Bibliothèque canadienne-française ", p. 169).

5 Voir F. Ouellet, «Se faire Père. L’œuvre de Daniel Poliquin ", loc. cit., p. 103106.

$6 \quad$ Ibid., p. 175. 
montre un fils incapable de se faire père, l'œuvre de Poliquin n'a guère progressé. Je reviendrai rapidement sur ce point en conclusion.

\section{Le refus de la paternité instituée}

Le début de Temps pascal nous présente donc Médéric Dutrisac, qui a dans la soixantaine, au moment où, en tant qu'il se donne à lire symboliquement comme un père déchu ${ }^{7}$, il est sur le point de refaire sa vie. Mais pour comprendre la déchéance de Médéric comme père déchu, il faut se donner une vision d'ensemble de son trajet et le saisir d'abord comme fils visant pour soi la paternité symbolique. Cette visée se trouve inscrite essentiellement dans un parcours signifiant qui interpelle l'autorité ecclésiastique. Ainsi, une suite de "pères " de l'Église jalonne le parcours du personnage.

L'avenir de Médéric prend forme lorsqu'il se démarque comme élève particulièrement doué du curé Berthier, lequel souhaite qu'il lui succède un jour. Cependant, au collège, qu'il fréquente moins par conviction religieuse que par obéissance aux voux formulés à son égard par le curé Berthier, Médéric fait la rencontre du père Rivard, qui lui propose plutôt de s'orienter vers l'enseignement. Auprès du père Lebel, à qui Médéric a été recommandé, il devient instituteur dans son village natal. Médéric a alors dix-huit ans. Il habite avec sa famille. Il a en quelque sorte la stature d'un père, par l'importance qu'il acquiert pour sa famille sur les plans de la réussite sociale et économique (car c'est ni plus ni moins qui pourvoit aux besoins de la famille, dont le père, Siméon Dutrisac, est impotent). Son parcours vers la paternité symbolique paraît ainsi tout tracé ; il ne lui reste plus qu'à faire honneur aux paroles du père Rivard : "Et puis, un jour tu te marieras, tu auras des enfants, tu seras un bon père de famille... ${ }^{8}$.

7 Symboliquement, parce que le personnage est bien entendu une figure textuelle. «Si donc le personnage littéraire n'a ni corps ni psychisme (et on n'en saurait démordre), force est de constater que ce vide esrt extraordinairement rempli, du fait même que le personnage est un signe. Ce que l'être fictif ne possède pas, le langage le lui prête dans l'esprit où il en fait émerger la représentation ", comme le dit si bien Raymond Joly ("L'auteur et ses personnages ", Le Pharsamon de Marivaux ou comment sinventer un sexe, Paris, PUF, 1995, p. 76).

8 D. Poliquin, Temps pascal, Montréal, Pierre Tisseyre, 1982, p. 54. Dorénavant, j'indiquerai la pagination entre parenthèses directement après la citation. 
Médéric est en place depuis peu lorsque le vicaire Robitaille, prenant la relève du père Rivard, lui conseille de se marier. Il précise : " tu as de meilleures chances de devenir principal d'école en étant marié'» (81). Le mariage, qui fonctionne métonymiquement en regard de la question du signifiant paternel (la femme importe ici dans la mesure où elle permet de fonder une famille), est le signe le plus tangible sur quoi fonder la paternité symbolique. S'il ne suffit pas d'avoir des enfants pour être père, il est vrai que, très souvent, la volonté de paternité symbolique prend appui sur la paternité biologique. Depuis sa rencontre avec le curé Berthier, Médéric a gravi assez facilement les échelons du système pédago-clérical. Cette foisci, la parole du vicaire Robitaille le fait réfléchir : « Médéric, rêveur, l’avait écouté sans comprendre. Tout d'un coup, il n'avait plus envie de se marier, ni de la place de principal, juste de ne rien faire » (81). Poliquin construit toujours l'identité de ses personnages en fonction de l'image que les autres nous renvoient de nous-mêmes9. Dans ce cas-ci, Médéric, pour la première fois, résiste devant le discours que les autres tiennent sur lui, comme s'il avait atteint la limite du parcours symbolique non pas qu'il vise, mais que les autres visent pour lui. Devenir père, cela signifie fondamentalement qu'on s'inscrive dans un parcours de paternité qui soit résolument différent de celui qu'offre le modèle contre lequel on se révolte. Cela est essentiel, le fils ne peut espérer prendre sa place dans la société que s'il se positionne dans un rapport de force vis-à-vis de la figure du père instituée. Dans ce contexte, le modèle de la paternité symbolique (et le contexte socioculturel que ce modèle engage) est incessamment à reconstruire, à redéfinir ${ }^{10}$. Ainsi, il aura suffi que Médéric parvienne presque à réaliser le parcours symbolique qu'on lui avait tracé pour que le texte déconstruise ce parcours en le réorientant de façon à placer Médéric en opposition avec ceux qui ont tenté de l'insérer dans leur propre discours. Avec les années,

9 Voir sur cette question F. Ouellet, "Une esthétique de l'identité en construction ", préface à D. Poliquin, Nouvelles, op. cit., en particulier p. 7-18 (en particulier p. 14-16) ; et L. Hotte, "Entre l'Être et le Paraittre : conscience identitaire et altérité dans les ouvres de Patrice Desbiens et de Daniel Poliquin ", dans Croire à l'écriture. Études en littérature québécoise en hommage à Jean-Louis Major, sous la dir. de Y. G. Lepage, Orléans, éd. David, 2000, p. 163-178 (en particulier p. 171).

10 J'ai tenté d'illustrer cette structure en recourant à la figure mythique de Moïse dans Passer au rang de Père. Identité sociohistorique et littéraire au Québec (Québec, Nota bene, 2002, p. 17-20).

Ouellet 49 
la révolte de Médéric prendra une forme toujours plus revendicatrice, essentiellement contre la domination anglophone dans le réseau scolaire et contre l'inhumanité de la morale religieuse. Si son plaidoyer en faveur du français témoigne déjà d'une réorientation radicale de la paternité symbolique qui est visée (une paternité politiquement à gauche), dont la viabilité est d'ailleurs éloquemment exprimée par " une certaine notoriété » (128) que lui apporte cet événement, sa révolte culmine dans une opposition ouverte contre le curé Viau (cinquième membre du clergé qui, à la suite du curé Berthier, des pères Rivard et Lebel et du vicaire Robitaille, intervient pour orienter la vie de Médéric). Ce dernier événement amènera Médéric à démissionner, cautionnant la rupture définitive avec le référent religieux ; ce qui du coup relègue symboliquement Médéric au rang de fils et du coup élimine à court terme le rêve de Médéric de «se bâtir à côté de la maison paternelle, une maison à lui pour ses propres enfants » (126). De fait, Médéric abandonne bientôt femme et enfants, l'échec conjugal paraissant confirmer l'échec d'une paternité symbolique qu’il n'avait jamais tout à fait atteinte et redonner à Médéric la liberté dont il avait besoin pour exprimer sa révolte vis-à-vis de l'autorité et s'engager, en tant que fils, dans un nouveau parcours de paternité : c'est en effet à la suite de ces événements qu'il militera dans le syndicalisme et qu'il s'engagera dans la politique, parvenant à se faire " un nom partout où il était passé dans le Nord de l'Ontario" (11).

C'est exactement ce parcours signifiant que Poliquin réintroduira dans L'Obomsawin, où le personnage éponyme offre une résistance vis-à-vis du modèle de paternité que lui offrent les fondateurs de Sioux Junction : après avoir peint des fresques historiques célébrant ses ancêtres, l'Obom se tournera vers la peinture amérindienne, qui le rendra célèbre.

\section{Le point de vue du fils lésé}

C'est ainsi que Médéric, surgissant du bois au début du roman, offre l'image du père déchu. Médéric n’est plus fils depuis longtemps (l'étape de la révolte est passée), mais ni tout à fait père : nous savons que sa révolte contre l'autorité ecclésiastique l'a engagé dans un nouveau parcours, mais ce trajet a échoué ; la dépression qu'il a faite au point de vouloir mourir est d'ailleurs la conséquence directe de cet échec. Or, le roman commence exactement sur la nouvelle occasion qui est offerte à Médéric de devenir père. Le texte conceptualisera ce nouveau parcours symbolique en fonction des personnages de Léonard et de Jacinthe. 
Revenons à l'incipit en citant les premières lignes du roman : " Jacinthe ouvrit les yeux et les referma aussitôt sans comprendre. Le coup de frein avait surpris les passagers dans leur sommeil. L'autobus s'était vraiment arrêté. [...] On allait parler de panne lorsque la porte s'ouvrit pour laisser monter un homme vieux et grand, avec des raquettes sur le dos. Sa barbe et ses sourcils étaient hérissés d'aiguilles de glace blanche " (5). L'image étonnante d'un Médéric Dutrisac vieilli, qui surgit de la nuit et de l'hiver au détour d'une route du nord, est digne d'une apparition. Le roman s'ouvre sur le regard de Jacinthe, une jeune fille d'une vingtaine d'années ; un regard endormi, entre le sommeil et l'éveil, qui traduit bien à la fois la présence réelle de cet homme et l'espèce de légende qui court à son sujet. Bien que Médéric se soit installé à ses côtés, Jacinthe ne le regarde pas, préférant penser à lui, à tout ce que son nom évoque, car dans sa famille on parlait souvent avec fierté de Médéric Dutrisac. Bientôt l'autobus est arrêté par des piquets de grève, et Médéric choisit de descendre pour poursuivre sa route à pied. Jacinthe descend aussi et, pour la première fois, pose enfin son regard sur Médéric et l'interpelle. Médéric lui répond : «Tu me connais, toi? " (13). Lui qui est si vieux qu'on a "oublié [son] âge depuis longtemps " (56), voilà qu'un regard de femme se pose sur lui et le reconnaît, c'est-à-dire distingue en Médéric l'image du chef syndicaliste et de l'ancien maire communiste. Bref, elle nomme ce père symbolique qu'il a été pour des générations de francophones bafoués.

Il faut ici ouvrir une parenthèse pour bien comprendre comment fonctionne la dynamique du signifiant paternel, beaucoup plus complexe qu'il n’y paraît. Poliquin, comme tout romancier, n'écrit jamais ses textes à partir du point de vue du père, mais toujours à partir du point de vue du fils. Si le roman focalise sur la figure paternelle (bien que problématique) de Médéric, c'est moins pour montrer sa difficulté à construire la paternité symbolique que pour le définir a posteriori comme figure de père possible vis-à-vis du fils-narrateur, Léonard Gouin, qui est le personnage principal ${ }^{11}$. C'est en fonction de celui-ci qu'il faut saisir la question du père dans Temps pascal; mais le roman est ainsi construit qu'il nous oblige d'abord à nous intéresser à Médéric. D'ailleurs, ce n’est que vers la fin que nous comprendrons que Léonard est le narrateur, comme si Poliquin avait eu de la difficulté à faire assumer le texte par le point de vue du fils. Bref, père

11 Ce que confirme Poliquin dans l'entretien publié dans Voix et images (F. Ouellet, "Le roman de "l'être écrivain" entre l'anonymat et la reconnaissance. Entretien avec Daniel Poliquin », loc, cit., p. 405). 
" incertain " pour lui-même12, Médéric est toutefois une imposante figure de père pour autrui, en l'occurrence pour Léonard, qui se trouve en position de fils symbolique dans la structure signifiante du texte. Cinq ans après Temps pascal, nous retrouverons la même structure dans L'Obomsawin, où le "personnage principal " n'est pas l'Obom, mais le

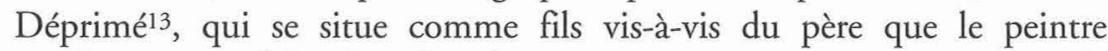
représente pour lui. C'est dans la rencontre entre ces personnages, par le biais de Léonard (j'y viendrai bientôt), que le roman se complexifie et qu'il fait apparaître la valeur (en termes d'éthique) de la représentation du signifiant paternel chez Poliquin. Invariablement, l'œuvre de Poliquin manifeste une volonté de redéfinir la verticalité des rapports. C'est bien cette verticalité que Médéric a remise en question en cherchant à conceptualiser, par son engagement social, une nouvelle forme de rapport à la loi. Qu'il n'y soit pas tout à fait parvenu est d'abord significatif de la signification de cet échec pour le personnage lui-même. Si Médéric échoue, c'est avant tout parce que lui-même, en tant que père, ne réussit pas à être à la hauteur de la forme horizontale qu'il essaie de concevoir pour définir le rapport à l'autorité. Or, c'est cette ambiguïté qu'incarne la figure de Médéric, et en tant qu'elle se trouve définie par rapport à la figure de Léonard, que le texte problématise en introduisant la figure de Jacinthe.

Car la relation de Médéric à Jacinthe aura pour conséquence de le faire échouer à réhabiliter la figure paternelle dans sa valeur horizontale. Revenons une fois encore à l'incipit. Par la reconnaissance de Jacinthe, le texte cherche à refonder la figure paternelle de Médéric. Reste à Médéric à retourner à Jacinthe son propre regard de reconnaissance. Ce qu'il a déjà fait en acceptant qu'elle fasse la route à pied avec lui ; ce que surtout il confirmera, quelques heures plus tard, en passant la nuit avec elle dans une chambre de motel de Sudbury. Cette relation ramène Médéric trente ans en arrière : "Pour un moment, il oubliait tout ce que le nom de Médéric Dutrisac résonnait de vieux. On était comme au temps où on sortait du bois après le chantier pour prendre un coup et courir les guidounes. Jacinthe n'était pas une guidoune, et Médéric n'avait pas dravé depuis trente ans, qu'importait, on se sentait bien comme ça " (8). Auprès de

12 L'on ne deviendrait jamais père que pour les autres, jamais pour soi. C'est Lacan qui disait, à ce propos : "Si tant est que pour chaque homme l'accession à la position paternelle est une quête, il n'est pas impossible de se dire que, finalement, jamais personne ne l'a vraiment été complètement " (J. Lacan, Le séminaire IV. La relation d'objet, Paris, Seuil, 1994, p. 205).

13 Voir ibid., p. 406. 
Jacinthe, Médéric a le sentiment de revenir trente ans en arrière, c'est-à-dire au moment où, après avoir démissionné de son poste d'instituteur et abandonné sa famille, il se lance dans le syndicalisme et se forge graduellement la réputation que l'on sait. Scène centrale, donnée en analepse, que le narrateur a significativement intercalée entre la rencontre dans l'autobus et le regard de reconnaissance posée par Jacinthe sur Médéric à la sortie de l'autobus. La structure narrative témoigne elle-même de l'importance capitale de la scène, l'emboîtant entre l'échec d'un certain désir de paternité et la reconnaissance possible de ce désir.

Or, ce regard de reconnaissance du père sur la femme, de Médéric sur Jacinthe qui pourrait être sa fille, c'est un regard coupable. Coupable parce que le roman conceptualise la figure de Médéric, sur le plan de la structure signifiante qui construit la représentation du signifiant paternel, en fonction du fils symbolique, Léonard. Le regard de Médéric sur Jacinthe est le regard de qui refuse de céder sa place au fils. Posséder Jacinthe, c'est ne pas savoir passer le relais au fils, dont le tour est venu de se faire père ${ }^{14}$. C'est précisément pour conceptualiser cette représentation œdipienne que le roman, je le répète, introduit le troisième personnage fort du roman, le fils-narrateur. Dans L'écureuil noir, le professeur Pigeon (ce personnage figure d'abord dans Visions de Jude), qui est en posture de père symbolique, saura dire à Calvin Winter : je vous présente Maud Gallant ${ }^{15}$. Nous

14 J'entends « ne pas savoir » dans le sens que lui donne Menaud, maître-draveur. Commentant le fameux " je suis d'une race qui ne sait pas mourir " du personnage éponyme, Ruggero Campagnoli met en évidence le rapport de force qui oppose Menaud et son fils : « Ne pas savoir mourir ne signifie pas savoir vivre, mais plutôt le contraire, dans une idéologie qui demande aux vieilles générations de savoir mourir pour céder aux nouvelles leur place et le droit de changer la vie " (Ruggero Campagnoli, « Menaud, le père qui ne sait pas mourir ou le syndrome québécois », Revue d'histoire littéraire du Québec et du Canada français, no 13, hiver-printemps 1987, p. 104).

15 C'est Poliquin lui-même qui a insisté sur cette " passation " du père au fils : «Pigeon est d'abord un pédant, un mauvais poète, puis il fait un retournement sur lui-même, une mutation, ce qu'on doit faire tous dans la vie. Alors lui le fait, et c'est là qu'il gagne la partie de bras de fer avec Jude, lui qui a toujours été battu par le fils, Jude lui ayant piqué la femme qu'il aimait [dans le roman Visions de Jude]. Le père gagne à la fin! Dans L'écureuil noir, la transformation est déjà faite, c'est un personnage qui n'a plus rien à espérer ou à attendre, qui est authentique cette fois. Il peut dire maintenant : Calvin, je vous présente Maud Gallant, car il n'est plus en compétition. Il est lui-même » (F. Ouellet, 
sommes bien loin de cet échange dans Temps pascal. Nous savons que les trois personnages seront très liés pendant quelques mois, mais Médéric ne saura pas céder Jacinthe à Léonard.

Au mieux esquisse-t-il un geste de bonne volonté en ce sens, et encore ce geste est justement déterminé par le sentiment de culpabilité s'y rattache. Nous sommes alors à la fin du long séjour des trois personnages au camp de Médéric, et Jacinthe prononce cette phrase fatale: « Je partirai après-demain. [...] Tout d'un coup, quelques mots anodins comme "je partirai après-demain" avaient suffi à changer la vie » (136). La parole de Jacinthe " change la vie » moins par la séparation qu'elle introduit qu'en fonction de la prise de conscience, par Médéric, d'une faute qu'il aurait commise à l'égard de Philippe. Aussi, le jour du départ de Jacinthe, Médéric dit à Léonard : «Fais donc ta valise toi aussi. Je pense que la petite aurait de la peine à voyager seule. Ça lui ferait du bien d'avoir quelqu'un à côté d'elle. Tu reviendras quand tu pourras." Léonard accepta tout de suite. En rougissant, les yeux à terre, il ajouta qu'il avait des choses à faire en ville » (137). Bien piètre lecteur celui qui n'y lirait pas, comme dans la relation entre le professeur Pigeon et Calvin Winter, la passation symbolique du pouvoir entre le père (faussement débonnaire) et le fils (gêné). Mais quelle misère ici! Car nous savons pourtant que Médéric, les ayant reconduits à l'autobus qui les amènera en ville, se saoule jusqu'à ne plus être en état de les voir partir, et qu'un an plus tard Jacinthe se suicidera... Il est vrai que nous sommes douze ans avant L'écureuil noir.

\section{Une éventuelle réconciliation}

La fin du roman tente de réconcilier le père et le fils — celui que le père a sacrifié à son désir pour la femme. Le roman se termine sur la possibilité qui est offerte à Médéric de se racheter auprès de Léonard, sans que le roman donne à lire l'expression même de ce rachat. À l'annonce de la mort de Jacinthe, la première réaction de Médéric a été d'aller retrouver Léonard à Ottawa, qu'il n'avait pas revu depuis leur séparation au Lac-à-la-Loutre. Il surprend Léonard chez lui en pleine nuit, lequel est précisément dans une période creuse : tout un pan de sa vie, des projets, des rêves, viennent de s'écrouler. Médéric ne pouvait pas survenir à un meilleur moment, comme si le texte rejouait la soudaine apparition du personnage dans

"Daniel Poliquin : L'invention de soi ", entretien, Nuit blanche, n 62, hiver 1995-1996, p. 57). 
l'incipit, mais cette fois-ci à la faveur de Léonard (au lieu de Jacinthe). En outre, c'est le jour de Pâques. C'est ici que le titre du roman émerge, acquiert sa signification. La fête de Pâques apporte une coloration symbolique religieuse à une rencontre capitale à un moment bien précis. Médéric et Léonard se retrouvent sans Jacinthe pour la première fois, c'est le jour de la résurrection du Christ, qui est mort à la fois pour racheter les péchés des hommes et leur montrer comment vivre en communauté, comment s'aimer les uns les autres. Cette leçon traduit exactement le défi qui attend Médéric auprès de Léonard, sauf que c'est son propre péché que Médéric doit racheter. La culpabilité est du côté de celui qui tient lieu de père aux yeux du fils. Médéric est « le Sauveur " pour Léonard : " Médéric, c'est toi Médéric, t’es venu m’aider, Médéric, le Bon Dieu est bon. Entre Médé, entre, je savais que quelque chose se passerait. Et te voilà, entre que je t'embrasse !" Médéric [...] était accueilli comme le Sauveur" (113).

Pour Médéric, voici venu, après le temps coupable (le temps du châtiment), le temps pascal : le rachat, la résurrection, la possibilité non pas exactement de se faire père, mais de se placer moralement dans des conditions qui le rachèteront et qui lui permettront peut-être un jour de se faire père auprès de Léonard, fils symbolique. En revanche, Léonard a la chance de trouver là un père ; celui en qui l'on peut croire, qui permet de croire. Le texte se trouve de la sorte à réinvestir, sur de nouvelles bases constructives, l'isotopie religieuse qui avait jadis canalisé la révolte de Médéric. Le roman rejoint ici la psychanalyse, où le lien religieux se forme à partir de la mise en place de la métaphore du Nom-du-Père. Il s'agit là de croire impérativement et intransitivement, parce que la croyance est affaire de structure signifiante. Or, Léonard est précisément croyant. Il a une " collection d'objets religieux » chez lui (33) et il assiste à la messe régulièrement, changeant d'église et de confession au gré de son humeur : "Il ne va pas toujours à l'église Sacré-Cour de la rue Laurier. Parfois, il va plutôt chez les Luthériens, ou chez les Anglicans ailleurs en ville, pour faire changement. Il se rend même quelquefois à la synagogue où il compte rencontrer Miss Krantzberg qui lui résiste toujours » (33). Le lieu et la religion importent donc relativement peu : ce qui compte, au-delà d'une ouverture qui traduit parfaitement l'ouverture et la nécessité de la quête de Léonard, c'est de croire. Auprès de son ami Philippe, Léonard soutenait déjà « que l'Église était sur le chemin de la vraie foi, que celle-ci ferait jour tôt ou tard et que lui, Léonard, serait témoin du Miracle de la Charité " (33). Le "Miracle de la Charité », c'est justement la promesse qu'offre la "venue » de Médéric, c'est l'exaltation du partage au lieu de la possession contre autrui, c'est l'idée dominante de l'égalité et de la fraternité entre les 
peuples, entre les cultures, entre les religions - et au premier rang entre le père et le fils aussi.

Car toute cette isotopie religieuse fonctionne comme un réseau métaphorique qui alimente une certaine relation au père, récurrente chez Poliquin. L’apparition salvatrice de Médéric, c'est un nouveau Médéric, qui se rachète, différent de celui qui a privé Léonard de Jacinthe, le fils de la femme. La foi de Léonard n'est possible qu’à ce titre. Auprès de Médéric, la foi du fils va se construire; elle est à l'image de «l'église du Sacré-Cour en reconstruction " (161) sur laquelle s'attache le regard de Léonard dans sa promenade qui clôt le roman. Temps pascal montre très bien comment fonctionne la dynamique signifiante eu égard à la valeur éthique qui la caractérise. L'origine de la révolte de Médéric, à partir de laquelle il s'était en tant que fils, trente ans plutôt, placé sur la voie de la paternité symbolique, était une révolte contre l'Église, plus précisément une révolte contre la parole autoritaire radicale, où la rhétorique du péché fonde la conscience coupable. Dès lors, il est logique que la réhabilitation du personnage, l'amorce de l'expression signifiante d'une déculpabilisation, réactualise la référence religieuse, mais en regard d'un nouveau modèle de paternité symbolique : un modèle fondé sur l'horizontalité des rapports plutôt que sur la verticalité. Médéric avait d'abord cherché en vain à convertir le rapport vertical en relation horizontale par son engagement communiste et syndicaliste ; la fin du roman suggère plutôt la réactualisation du lien religieux, afin que l'horizontalité des rapports ne soit pas complètement dépourvue de spiritualité.

Dans L'Obomsawin, Poliquin fera évoluer la relation entre l'Obom et le Déprimé afin de parvenir à établir un rapport d'égalité cependant débarrassé des références religieuses ${ }^{16}$. Il reste que la figure christique va opérer un retour en force avec le personnage de Benjamin Saint-Ours, Poliquin à la fois retrouvant l'écriture de Temps pascal et rejoignant la tendance de l'écriture romanesque des années $1990^{17}$. La métaphore du Christ est centrale : c'est par elle que s'effectue le passage de la verticalité

16 Encore que l'Obom décède assis sur le banc d'église sculpté par son ancêtre, Byron Miles, qui sur la fin de sa vie avait retrouvé la foi anglicane.

17 Sur cette question, voir F. Ouellet, " Le nouveau roman québécois et la métaphore christique : fragments d'un discours amoureux ", Laval théologique et philosophique, vol. 59, no 3, octobre 2003, p. 451-461 ; F. Ouellet, " Le fils de l'autre. L'intertexte christique et la figure du Père dans Incarnations d'Emmanuel Aquin ", Études francophones, vol. 18, no 2, automne 2003, p. 135-147.

\section{6 * LittéRéalité}


du IL du Dieu vengeur de l'Ancien Testament vers l'horizontalité du TU dans le Nouveau Testament.

\section{Conclusion}

Je reviens à l'incipit de Temps pascal et à l'excipit de L'homme de paille que j'ai évoqués en introduction. La différence est ici que Médéric Dutrisac est une figure de père déchu, tandis que Benjamin Saint-Ours est une figure de fils qui cherche désespérément à devenir père. À cet égard, Benjamin est surtout proche de Léonard, un peu comme si l'écriture de Poliquin, après le progrès que permettait de consigner L'écureuil noir quant à la représentation de notre problématique, tendait à revenir au point de départ. Dans tous les cas, la relation du père et du fils qui termine Temps pascal, qui fait la promesse d'une réconciliation exaltante qui débouche de part et d'autre sur la paternité symbolique, nous l'attendons encore. Dans le deuxième roman de l'auteur, l'Obomsawin tient lieu de père symbolique auprès, une fois de plus, du narrateur, en l'occurrence le Déprimé. Lorsque l'Obom meurt à la fin, le Déprimé est sur le point de refaire sa vie. La situation de Jude, héros de Visions de Jude, n'est pas plus heureuse. Il vit seul et tente, lui aussi, de refaire sa vie. Tout lui a réussi, sauf l'amour. Cependant, le héros de L'écureuil noir s'est pris d'affection pour un jeune garçon dont il pourrait être le père et fait des projets de vie commune avec Maud Gallant. Or, il semble, avec L'homme de paille, que tout soit à reprendre au début - peut-être en raison de l'introduction de l'Histoire dans le roman. Outre que Benjamin est sans père, il fait des enfants malgré lui (il est dans le coma!). On ne peut guère trouver, je pense, de situation plus symptomatique d'une impuissance insistante à devenir père. 